\title{
Dinámica emprendedora en el cultivo aloe, estado Falcón, Venezuela
}

\author{
Piña Zambrano, Henri * \\ Villasmil, Carlos ** \\ Morales, Agustín***
}

\section{Resumen}

El aloe es una planta con una vasta tradición en Falcón (Venezuela), sin embargo aún se continúa explotando de manera artesanal. Estas condiciones se suman a la ausencia de una agroindustria generadora de bienes finales de alto valor agregado, tal como lo demanda el actual mercado. Tal situación conlleva a considerar esta actividad como marginal, desaprovechando significativas potencialidades para construir un conglomerado agroindustrial en torno a este cultivo. Partiendo de estas premisas se condujo una investigación para analizar la dinámica emprendedora en el cultivo aloe, enfatizando la interpretación de las iniciativas de emprendimiento desde la perspectiva del perfil emprendedor y del tipo de empresa constituida. La metodología se centró en un estudio de tres niveles de análisis: productores, procesadores artesanales y agroindustria, a quienes se les aplicó un cuestionario. Los resultados evidencian una dinámica emprendedora limitada y concentrada en la fase de producción primaria, con escasas iniciativas agroindustriales y menos aún, en el sector de elaboración de bienes finales. Se impone la construcción del andamiaje institucional favorable para la promoción de iniciativas de emprendimientos, así como el manejo de información de mercado e innovación tecnológica, como elementos claves de dichos emprendimientos.

Palabras clave: Aloe, emprendimiento, conglomerado, estado Falcón, Venezuela.

\section{Recibido: 28-03-12. Aceptado: 15-06-13}

* Ingeniero Agrónomo, MSc. Gerencia Agrícola. Doctor en Planificación y Gestión del Desarrollo Regional (LUZ, Venezuela) y Doctorando en Desarrollo Rural (Universidad de Córdoba, España), profesor Departamento Desarrollo y Producción Agrícola, Universidad Francisco de Miranda, Venezuela, e-mail: henripina@gmail.com

** Ingeniero Agrónomo, egresado del Programa de Ingeniería Agronómica de la Universidad Francisco de Miranda, e-mail: c.villasmil84@gmail.com

*** Profesor titular. Departamento Instituto de Economía Agrícola y Ciencias Sociales, Universidad Central de Venezuela. Coordinador Unidad de Investigación Agroalimentaria Facultad de Agronomía, e-mail: moralesa@agr.ucv.ve, amauta_ve@yahoo.com 


\title{
The Entrepreneurial Dynamic in Aloe Vera Cultivation, State of Falcon, Venezuela
}

\begin{abstract}
Aloe vera is a plant with a vast tradition the State of Falcon, Venezuela; nevertheless, it continues to be exploited as a cottage industry. These conditions are due to the absence of an agroindustry that generates final products of high added value, which the current market demands. Therefore, this activity is considered marginal and advantage is not taken of significant potentials for constructing an agroindustrial conglomerate around this crop. Starting from these premises, research was carried out to analyze the entrepreneurial dynamic in aloe cultivation, emphasizing the interpretation of entrepreneurial initiatives from the perspective of the entrepreneurial profile and the type of enterprise that is established. The methodology centered on a study of three analysis levels: producers, cottage industry processors and agroindustry, to whom a questionnaire was applied. Results showed a limited entrepreneurial dynamic concentrated in the primary production phase, with scarce agroindustiral initiatives and even fewer in the final products sector. The construction of institutional scaffolding favorable for promoting entrepreneurial initiatives as well as handling information about marketing and technological innovation are required as key elements for such enterprises.
\end{abstract}

Keywords: Aloe vera, enterprise, conglomerate, State of Falcon, Venezuela.

\section{Introducción}

Los procesos globales de desarrollo conllevan la posibilidad de ubicar en cualquier lugar del planeta, proveedores especializados para soportar procesos productivos innovadores, incluso en apartadas regiones del planeta. De esta acción deriva como principal consecuencia, la pérdida de capacidad competitiva de agentes locales, si éstos carecen de las destrezas requeridas por las empresas demandantes de sus insumos.

En este sentido y aunque no todos los territorios son agronómicamente aptos para cultivar aloe, el procesamiento y elaboración de bienes finales pueden localizarse en cualquier lugar del mundo, atendiendo a la viabilidad y factibilidad de tal proceso. Por tanto, es importante dejar en claro la real existencia de otros territo- rios con condiciones económicas, sociales o políticas más apropiados a los de Falcón para la siembra de aloe, por lo cual se convierten en potenciales áreas futuras de inversión, compitiendo directamente con una actividad arraigada en la historia del estado, pero ante la actual dinámica mundial, ha exhibido una pobre capacidad de respuesta orientada a un mayor nivel de competitividad y hoy día está amenazada por el surgimiento de nuevos enclaves de producción.

Desde este punto de vista, es claro el significativo efecto del emprendimiento en respuesta al grado de integración de iniciativas en torno a una actividad en común (Presutti y Boari, 2008), convirtiéndolo en un factor determinante en la promoción de nuevas iniciativas productivas, en respuesta a los beneficios generados por las economías de aglomeración, es- 
cala y ámbito surgidas a partir de la conexión entre emprendimientos y favoreciendo la identificación de nuevas oportunidades de inversión en su zona de influencia (Romero y Montoro, 2008).

Sobre la base de los planteamientos anteriores, se presume la presencia en diversas localidades de Falcón (territorio) de un patrón evolutivo sustentado parcialmente en un legado histórico (patrimonio natural propio) de recursos (sociales, económicos y culturales), los cuales han favorecido la siembra y explotación del aloe en Falcón a lo largo del tiempo. Sin embargo, se considera un escaso aprovechamiento de tal patrimonio, desperdiciando una significativa oportunidad para establecer diversos emprendimientos en torno al aloe en el estado. Igualmente, se presume que los agentes económicos presentes en el cultivo no han identificado adecuadamente las exigencias de su mercado (aprovechamiento de oportunidades), por lo cual no han existido estímulos orientados a desarrollar iniciativas emprendedoras para satisfacer las necesidades exigidas por la demanda.

Dentro de este contexto, la sistematización productiva de zonas de explotación de aloe en Falcón, pueden llegar a generar procesos de crecimiento y desarrollo al articular, organizada y coherentemente, sus actores y redes a objeto de crear un ambiente proclive hacia externalidades positivas, para generar e implementar innovaciones tecnológicas y nuevo conocimiento, partiendo del saber hacer local acumulado en el transcurso de la evolución histórica del cultivo en el estado, como elementos promotores de iniciativas emprendedoras dentro del territorio.

En función de lo anterior, se planteó como objetivo de esta investigación analizar la dinámica emprendedora en el cultivo de aloe vera, haciendo énfasis en la creación de esos emprendimientos desde la perspectiva del perfil emprendedor y del tipo de empresa constituida. Desde el punto de vista metodológico, la investigación tuvo un carácter descriptivo-explicativo-analítico, teniendo como unidad de estudio al cultivo aloe en Falcón (Piña y Morales, 2010; Piña, 2006), delimitada a aquellos municipios donde dicha planta es sembrada y explotada, por lo cual su alcance se circunscribe a esta porción geográfica de territorio.

Estas son zonas caracterizadas por sus condiciones de marginalidad económica, llegándose en algunos casos a niveles de desempleo cercanos al $70 \%$ y cuya principal actividad se sustenta en la cría extensiva de caprinos, actividades comerciales menores y la eventual explotación de hortalizas de secano. Desde el punto de vista agroclimático, son de tipo semiárido: con hasta 9 meses secos, temperaturas promedio de $24^{\circ} \mathrm{C}$ y precipitaciones menores a $500 \mathrm{~mm} /$ año, por lo cual la evapotranspiración anual supera hasta 8 veces la precipitación. Los suelos varían desde textura arcillosa a franco-arcillosa y arenosos, con bajo contenido de materia orgánica. Poseen un drenaje externo moderado y permeabilidad de moderada a lenta. Están limitados por excesos de sales, moderada erosión y presencia abundante de calcio. La vegetación predominantemente es del tipo xerófita, característica del bosque muy seco tropical (Piña, 2004).

La muestra del estudio correspondió a tres niveles de análisis: productores, procesadores artesanales y agroindustria. A nivel de productores se abordaron 
las asociaciones responsables de la gestión de 6 plantas procesadoras promovidas por el Ejecutivo Regional, en la persona de sus juntas directivas. Complementariamente, se celebraron encuentros de trabajo tipo focus group con una muestra representativa de 60 socios pertenecientes a dichas asociaciones y cooperativas, de manera de conformar un perfil del fenómeno en estudio lo más acertado y fehaciente posible.

En el nivel de procesadores artesanales, se entrevistó a cinco individuos, quienes de manera informal elaboran bienes finales a base de aloe, ampliamente conocidos por la comunidad, pero que no están agrupados en ningún gremio en específico o registrados formalmente como empresarios (aunque no existe un registro oficial, se estima este número en cerca de 25 personas). Éstas son iniciativas informales con dilatada tradición en el estado y representan un segmento importante y reconocido, dentro del rubro aloe. Por su parte, el nivel agroindustria estuvo conformado por la procesadora de aloe ubicada en la $\mathrm{Pe}$ nínsula de Paraguaná.

En los tres casos, la información se obtuvo a partir de la aplicación de un cuestionario a través de una entrevista semiestructurada, en donde se destacó el perfil de emprendedor, perfil del emprendimiento y la visión actual y futura de tales iniciativas. Un primer momento de la fase de campo se realizó durante los meses de marzo-agosto de 2010, teniendo un segundo momento entre julio-septiembre de 2012, con el propósito de contrastar y verificar la información obtenida y procesada, con los agentes involucrados en el estudio.

\section{Algunas precisiones teóricas sobre emprendimiento}

Las categorías emprendimiento y emprendedor han sido ampliamente debatidas los últimos años desde diferentes puntos de vista y enfoques de análisis, enriqueciendo sustancialmente el debate sobre el tema. Originalmente ambos términos derivan del francés entrepreneur, el cual sin una acepción literal en idioma español, se ha utilizado como pionero y es más recientemente cuando se usa como emprendedor o emprendimiento. Tal situación ha dado origen a un sin número de definiciones, atendiendo al enfoque de estudio aplicado en cada caso.

Así, las concepciones del término incluyen desde características relacionadas con la innovación y la creatividad, hasta incluso asociar emprendimiento con gerencia y administración de empresas. Por tanto, la precisión y alcance del término estará dada por el contexto y objetivo a lograr en una investigación en particular, otorgándole un carácter multidimensional e incluso, ambiguo al concepto. Teniendo en consideración los anteriores aspectos y a pesar de esta amplia gama de enfoques, los principales postulados teóricos para explicar la existencia y persistencia de la actividad emprendedora se centran en 3 teorías básicas (Tíryakí, 2008).

La primera propuesta teórica corresponde a Marshall (1920), quien se centra en explicar cómo los mercados buscan el equilibrio bajo el supuesto de competencia perfecta. Para Marshall pequeñas contribuciones de un gran número de empresas de modestos emprendedores, lideran el progreso económico. Tal 
planteamiento se sustentaba en la existencia de información homogénea y transparente manejada por todos los agentes por igual, asimismo, no diferencia claramente el proceso de emprendimiento de un mecanismo de producción.

La segunda teoría corresponde al aporte de Kirzner (1997), quien conceptualiza al emprendedor como un individuo atento, vigilante del comportamiento del mercado y de las transacciones bursátiles o mercado de valores. Este autor diferencia conceptualmente conocimiento de información, al crear el primero, oportunidades para el potencial emprendedor. Su planteamiento central se sustenta en la existencia de una economía en desequilibrio la cual tiende al equilibrio gracias a las acciones de emprendedores alertas. Es una propuesta mecanicista donde en el proceso emprendedor no se toma en cuenta la experiencia personal y las lecciones del pasado.

El tercer aporte teórico es de Schumpeter (1927), quien redirige el análisis de mercado en equilibrio de Marshall y lo orienta hacia el desarrollo humano-económico, el cual es el resultado histórico de la continua destrucción creativa de los emprendedores, entendiendo a este agente, como un innovador y no como imitador de la producción. Schumpeter define emprendedor como quien establece un nuevo negocio o empresa para producir un nuevo bien, servicio o una mejora innovativa sobre otro ya existente. Lo diferencia del imitador por el uso de innovadores métodos de producción o por incursionar en nuevos mercados. Para este autor, el emprendedor toma la categoría de revolucionario al crear y po- ner en práctica nuevas funciones y métodos de producción, de allí su calificación de destrucción creativa.

A los efectos de esta investigación, se asume el concepto de emprendedor expuesto por Schumpeter, aunque igualmente se reconocen los postulados de Marshall: mercado en equilibrio y de Kirzner: individuo alerta. Sin embargo al contrastar la dinámica y entorno detectado en el cultivo aloe, se consideran insuficientes y limitados para explicar el comportamiento de sus agentes. Por una parte en el planteamiento de Schumpeter, destaca la posición del monopolio temporal detentado por el emprendedor al introducir una innovación en el mercado, lo cual resulta una situación evidente en este cultivo (Piña y Chirino, 2008).

Sin embargo, Schumpeter también destaca el descenso de esa posición de dominio en función del ciclo natural del negocio y la aparición de imitadores de la innovación introducida por el emprendedor e igualmente, es la posición de monopolio de donde se derivan las principales ganancias de dicho emprendedor (Schumpeter, 2003). Complementariamente, para este autor la innovación es la causa de desarrollo y el emprendedor es quien propicia los procesos de innovación, por tanto, se establece una relación de dependencia recíproca entre innovación como motor de desarrollo y emprendedor como generador y gestor de dichas innovaciones. En este sentido, no todos los individuos son emprendedores o innovadores, sino aquellos con la suficiente capacidad de reconocer oportunidades en su entorno (Schumpeter, 1947). 
Asimismo, es importante destacar como ambas posiciones: Schumpeter y Kirzner, pueden ser totalmente válidas en un momento determinado. Cada una será más pertinente para explicar el comportamiento emprendedor en la medida que se asuma estudiar el cambio generado por estos agentes en una situación particular. Para Schumpeter es la creación destructiva, donde el interés se orienta a la producción de innovaciones incrementales a objeto de modificar el equilibrio existente y promover en consecuencia, nuevos procesos y nuevas formas de elaborar bienes y servicios.

Por el contrario, sí el interés se centra en los cambios generados por el emprendedor desde el punto de vista interno en dicha situación, se estará frente al postulado de Kirzner: individuo alerta. En tal visión, el centro de atención se corresponde con un proceso de búsqueda e identificación de situaciones asimétricas o esquemas ineficientes de funcionamiento, a partir de los cuales puedan surgir oportunidades para los emprendedores atentos a tales variaciones del entorno. En este caso, la información sobre la evolución de los cambios es vital para la toma de decisiones por parte de los individuos atentos a su comportamiento.

Así, el desarrollo económico y, en consecuencia, el proceso innovador y emprendedor de un país será el natural resultado de la dinámica social, económica, política y cultural de una sociedad en un momento dado (Montoya, 2004). La dinámica evolutiva de la sociedad conformará el contexto para promover o restringir la innovación y el emprendimiento de la nación. Se crearán instituciones a tal fin (North, 1991).
Desde esta perspectiva, el enfoque institucional sobre el emprendedor, ubica el análisis más allá del plano meramente económico y lo sitúa dentro de un abordaje más social, sociológico; de tal forma, el proceso no puede entenderse adecuadamente fuera del contexto sociocultural, siendo importante resaltar lo significativo del ambiente social e institucional para el emprendedor. Por tanto, el proceso emprendedor se corresponde con la combinación interactiva de tres factores: emprendedor, individuo quien decide crear una actividad productiva; experiencia, forma en la cual se abordará el proceso emprendedor; y tecnología, medios disponibles para que el emprendedor aproveche esa oportunidad.

En términos concretos, una oportunidad de emprendimiento se concibe como aquella situación en donde atendiendo a las condiciones de mercado, se pueden introducir nuevos bienes, servicios o modos de producción y procesamiento. Esta definición está asociada al manejo de información por parte del potencial emprendedor, así como de su capacidad cognitiva para interpretar y procesar esa información (Shane y Venkataraman, 2000). En este sentido, el procesamiento de información, la creación de conocimiento, la innovación y la identificación de oportunidades están estrechamente relacionadas una a otra, siendo el aprovechamiento de la oportunidad, un atributo crítico del emprendedor.

En esta concepción de oportunidad de emprendimiento, destacan dos elementos clave: su carácter exógeno y la inclusión de la categoría tiempo en su concepto. Respecto a la primera, esta visión responde a la filosofía de Kirzner: oportu- 
nidades en el mercado. Sin embargo, es ampliamente conocida la vía endógena de creación de oportunidades a través de la combinación de talento humano y generación de nuevo conocimiento. En cuanto al tiempo, esta variable está implícita en el proceso mismo de identificación y aprovechamiento de la oportunidad de emprendimiento: se requiere tiempo para analizar y madurar la decisión de comenzar una nueva iniciativa de emprendimiento (Vaghely y Julien, 2010).

En este sentido, las oportunidades se corresponden con fenómenos objetivos: existen independientemente de los individuos, vigente mientras exista una necesidad para satisfacer. Sin embargo, es importante destacar el carácter y naturaleza objetiva y tangible de la oportunidad de emprendimiento, aunque su apreciación por parte de los individuos sea de carácter subjetivo e intangible. Por tanto, estas surgen principalmente de los mercados en desequilibrio con necesidades aún no satisfechas o cubiertas satisfactoriamente. Asimismo, las oportunidades de emprendimiento aparecen sustentadas en el manejo de la información asimétrica por parte de los individuos, shocks externos hacia el entorno local o variaciones en la oferta -demanda de bienes o servicios-(Plummer et al., 2007).

Una concepción como la anterior, está indisolublemente atada al carácter humano, en tanto social, de la actividad emprendedora. En consecuencia, la materialización del emprendimiento es un fenómeno personal, humano, social: en donde un individuo decide, atendiendo a su conocimiento, redes y capital social emprender una actividad de la naturaleza descrita (Grebel et al., 2001). Por lo cual, el acto de emprender, ergo, aprovechamiento de una oportunidad, depende de la evaluación personal de cada individuo sobre el particular. Así, el éxito de un emprendimiento está íntimamente asociado a los recursos individuales y la capacidad gerencial del emprendedor, los cuales a su vez están integrados en una combinación y complementariedad de destrezas y competencias. En otras palabras, la supervivencia del emprendimiento no es sólo un tema financiero o técnico (Grebel et al., 2001).

De esta manera y en concordancia con el planteamiento central de la investigación, enfocada en la dinámica emprendedora del circuito aloe, destaca el hecho de cómo las concentraciones geográficas de empresas responden básicamente a la estructura de las oportunidades para el emprendimiento existentes en una zona en particular, lo cual a su vez, se transforma en fuerza y motivación de reforzamiento y mantenimiento de esa concentración geográfica en un ámbito institucional normativo y regulador (Sorenson y Audia, 2000).

Es así como la densa concentración de empresas y nuevos emprendimientos favorece las opciones de potenciales emprendedores en esas zonas en particular, incrementando por tanto, la tasa de nuevos emprendimientos, lo cual a su vez fomentará la generación y difusión de nuevo conocimiento para la creación de nuevos emprendimientos, disponible a través de las redes sociales propias de cada individuo y a la capacidad de éste para procesar y aprovechar ese conocimiento (Park, 2005).

En otras palabras, la persistencia de concentraciones geográficas de em- 
presas responde a factores como la estructura de las oportunidades presentes para el emprendimiento como elemento aglutinador de dichas empresas en el conglomerado. Por otro lado, la integración de individuos en diferentes tipos de redes le proporciona un elemento clave en la creación de nuevas empresas, a través del acceso a información y conocimiento como insumo vital para la rentabilidad y éxito de la actividad emprendedora.

De esta manera, la participación en redes constituye un mecanismo para sortear vacíos estructurales y subsanar potenciales déficits de información, derivando en la aparición de oportunidades de inversión. Esto es: el emprendimiento está integrado y contextualizado a un entorno social el cual es influido, canalizado, limitado, inhibido y facilitado por su posición en la red social; en consecuencia, el emprendedor no puede considerarse un agente económico aislado.

En estos términos, la integración es un factor clave en el emprendimiento y aún más, en el área rural, vista sus características típicas de limitado acceso a recursos. A nivel local, el grado de integración está determinado por redes, lazos y relaciones del emprendedor. Así, las redes proveen el mecanismo para la integración. La reciprocidad en una integración le provee al emprendedor de conocimiento, relaciones y recursos, pero éstas sólo serán beneficiosas con la comprensión manejada por éste sobre el aprovechamiento de dicha oportunidad.

Por otro lado, en el proceso emprendedor, el ambiente local actúa como un contexto socioeconómico en el cual las relaciones sociales impactan en los productos económicos. Lo anterior es de vital importancia, porque si bien estas oportunidades pudieran haber estado siempre presentes, no se materializan para todos los individuos. Así la integración emprendedora crea una conexión entre la esfera social y económica del individuo (Jack y Anderson, 2002).

\section{Emprendimiento en el cultivo aloe Falcón}

En esta sección, se presentan algunas categorías que permitirán el análisis del emprendimiento del cultivo de aloe en el estado Falcón. En primer lugar se analiza el perfil del emprendedor, en segundo lugar la actividad productiva desarrollada por el emprendedor y, en tercer lugar la visión del emprendedor en cuanto a sus proyectos en un corto y mediano plazo.

\section{a. Perfil del emprendedor en el cultivo aloe Falcón}

El primer elemento de análisis de la investigación lo representa el perfil de emprendedor, aquel agente quien estructuralmente realiza los cursos de acción dentro del cultivo aloe. Dicho emprendedor se corresponde con una persona joven, cuya edad promedio es de 37 años (57\%), en quien existió desde temprana edad la inquietud e idea de incursionar en el procesamiento del cultivo, principalmente en el caso de los productores, por estar ligados tradicionalmente a su explotación familiar, pero siempre les fue cuesta arriba materializar una iniciativa de tal naturaleza. Estos agentes señalan que hasta en 3 intentos (individual o colectivamente) han promovido iniciativas en torno al rubro, todos infructuosos. 
En el caso de los procesadores artesanales, la inquietud por iniciar un emprendimiento dentro del rubro aloe tiene características marcadamente familiares y tradicionales: alguien del núcleo familiar inició la preparación de bienes finales, principalmente de tipo médico y cosmético, y pasó ese conocimiento a otros miembros de la familia durante varias generaciones. Por lo tanto, el procesamiento corresponde más a la puesta en marcha de un conocimiento ancestral que a la decidida y sistemática explotación de una oportunidad de negocio, correspondiendo con el carácter informal del emprendimiento.

Por el contrario, la agroindustria privada si se corresponde con el típico aprovechamiento de oportunidad de negocio. Partiendo de una exploración del sector, se detecta la posibilidad de incursionar en una actividad rentable, se realiza un detallado plan de negocios y se toma la decisión de invertir un capital en una idea analizada y ponderada.

En estos emprendimientos resalta la presencia masculina en su conformación $(80 \%)$, aunque sólo los procesadores artesanales son mujeres. Vale destacar, igualmente, la ausencia de mujeres en las juntas directivas de las asociaciones y cooperativas de productores, aunque las decisiones de emprendimiento se toman en asamblea general de socios, donde éstas participaron con su voto. Asimismo, la participación femenina en la actividad emprendedora en aloe, también está asociada a un rango mayor de edad (> 65 años), tal como es el caso de los procesadores artesanales.

El nivel de instrucción varía significativamente en las 3 modalidades analizadas. Los procesadores artesanales tienen en su mayoría una formación de primaria incompleta. En las asociacio- nes y cooperativas de productores se pueden encontrar desde analfabetas hasta universitarios en una amplia gama de especialidades: licenciados en educación, ingenieros agrónomos o administradores. Por su parte, en la mediana empresa son en su totalidad individuos universitarios con estudios de maestría en áreas afines a los negocios, finanzas o mercadeo.

Los padres de los entrevistados no ejercían una actividad asociada con el procesamiento del aloe, aunque en el caso de las asociaciones y cooperativas de productores, destaca una tradición en el cultivo y recolección de la planta. De éstos, $93 \%$ son empleados y sólo 7\% tienen un autoempleo. En este sentido, los padres no estuvieron ligado a iniciativas de emprendimiento de ningún tipo, por lo cual podría en principio, descartarse la influencia, patrón o rol de los padres en la decisión de los hijos a emprender en éste ámbito. En cuanto a las condiciones socioeconómicas, $60 \%$ de los entrevistados pertenece a la clase media, $13 \%$ media alta y $27 \%$ baja, atendiendo a su nivel de ingreso.

En este contexto, no se evidenció una relación directa entre actividad previamente desempeñada y motivación a emprender. Incluso en los procesadores artesanales ésta corresponde a un pase de testigo entre miembros del núcleo familiar. Por lo cual, se está frente a aquellos emprendedores por imitación y no ante el real aprovechamiento de una oportunidad de negocio detectada de manera sistemática. Sin embargo, tal como se recoge en la Tabla 1 , se confirma la relación directa entre clase social y acceso al sistema educativo (Kantis et al., 2004) (Tabla 1).

Los resultados anteriores terminan de ratificar (a excepción de la agroindustria privada) otro de los hallazgos de in- 
Tabla 1

Perfil del emprendedor: nivel de formación y experiencia previa

\begin{tabular}{lclc}
\hline Nivel de educación & $(\%)$ & Experiencia laboral & $(\%)$ \\
Primaria Completa & 7 & Agroindustria similar & 7 \\
Primaria Incompleta & 7 & Agroindustria distinta & 53 \\
Secundaria Completa & 53 & Actividad diferente al agro & 40 \\
Universitario & 33 & Sin experiencia laboral & - \\
\hline
\end{tabular}

Fuente: Elaboración propia.

vestigaciones anteriores (Piña 2006; 2005; 2004): el aloe no es la principal fuente de ingresos del núcleo familiar de aquellos agentes participantes con iniciativas productivas dentro del cultivo, lo cual se transforma en otro elemento clave a la hora de explicar la dinámica de los emprendimientos en este sector.

En otro orden de ideas, los agentes entrevistados $(87 \%)$ manifestaron no haber incursionado en otro tipo de emprendimiento, por tanto ésta era su primera experiencia. Aquellos casos que respondieron afirmativamente (13\%), lo hicieron en actividades comerciales de compra-venta de ropa y calzado, así como venta de productos por catálogo. Nuevamente, no son emprendimientos innovadores. Estos resultados muestran una baja disposición y proactividad al emprendimiento.

Una respuesta parcial a esta situación se encuentra en las limitaciones asociadas a las condiciones de las áreas rurales donde hacen vida la mayoría de estos agentes (salvo la agroindustria privada). Sin embargo, más allá de ese entorno desfavorable, también se distingue la motivación y vocación personal de cada individuo para incursionar en una iniciativa de emprendimiento, pero que al enfrentarse a la escasez de recursos y de opciones (a pesar de existir muchas ne- cesidades insatisfechas en ese medio) se abstienen de emprender cualquier iniciativa productiva.

Este comportamiento responde a una racionalidad asociada al tamaño del mercado y a la organización de la infraestructura donde debe apoyarse. Igualmente, la experiencia laboral previa tampoco resultó en un factor determinante para decidir incursionar en esta iniciativa productiva. Sólo 7\% manifestó haber laborado como empleado en una empresa relacionada con el aloe, mientras que el resto de entrevistados, labora en otros sectores distintos al cultivo y procesamiento del aloe. En contraste, la agroindustria privada tiene como principal fuente de ingreso la actividad relacionada con el aloe.

Derivado de lo anterior, casi todos los emprendedores (97\%) desconocían, más allá de la actividad de la producción primaria y salvo en el caso de los productores y procesadores artesanales, el proceso productivo del emprendimiento en el cual estaban incursionando, por tanto no estuvieron en contacto con las destrezas técnicas previas necesarias, requeridas y demandadas por la iniciativa a emprender.

Atendiendo a estos resultados, la principal motivación del emprendedor para incursionar en una actividad produc- 
tiva dentro del aloe, estuvo relacionada fundamentalmente con la mejora de la situación económica (40\%), junto a la conformación de un negocio propio $(30 \%)$, rentabilidad del negocio $(20 \%)$ y demanda de productos a base de aloe $(10 \%)$. Para las asociaciones y cooperativas de productores, este negocio propio lo asocian a mayor control del proceso productivo y a la paulatina eliminación de los intermediarios, con la consecuente mejora de los precios, el cual es señalado como la principal limitante a nivel de producción primaria de aloe.

Complementariamente, al consultarles sobre por qué decidió incursionar en esta actividad, $40 \%$ respondió que debido al atractivo mercado de los productos derivados del aloe. Sobre este particular, refirieron informaciones de algunos organismos oficiales que se hacían eco de aseveraciones acerca de la alta rentabilidad y los altos precios de los productos a base de aloe, sin embargo no mostraron seguridad y confianza sobre los clientes finales y los mecanismos de mercado para acceder a éstos. Su decisión de emprender estuvo sustentada en informaciones secundarias, incluso terciarias, no corroboradas en su totalidad. Este aspecto, aparece sin lugar a dudas, como uno de los elementos claves responsables de la actual situación de los emprendimientos a nivel de asociaciones y cooperativas de productores. El caso de los procesadores artesanales es diferente, dado la histórica relación con sus clientes.

Desde otro punto de vista, tanto el núcleo familiar como el entorno inmediato del emprendedor, estuvieron de acuerdo $(60 \%)$ y muy de acuerdo $(30 \%)$ con la idea de incursionar en una actividad de éste tipo. Así el apoyo recibido por los emprendedores por parte de familiares y amigos, se transforma en un factor crítico para continuar con su decisión de emprender, principalmente para los productores, quienes debían dedicar esfuerzos, tiempo e incluso dinero para llevar adelante una idea que no era del todo factible: dependía de muchos factores ingobernables, dando un significativo nivel de incertidumbre y en cierta modo, ambigüedad a su decisión. Estos recursos y esfuerzos, en extremo escasos y altamente valorados, podían utilizarse para otras opciones, sí bien menos rentables, pero sí, menos complejas y más seguras, lo que en definitiva contribuiría con el aumento del presupuesto familiar.

En términos generales, los emprendedores descartaron el impacto de sus iniciativas para contribuir con el crecimiento y desarrollo local como elemento motivador para emprender ésta, o el reconocimiento social a la idea de ser su propio jefe. Estas respuestas orientan la decisión de emprender, tal como ellos lo manifestaron, a la sentida necesidad de mejorar económicamente para satisfacer necesidades propias. Es necesario satisfacer necesidades propias, luego satisfacer ajenas, opinaron algunos de ellos.

En cuanto a la formación profesional específica de emprendimiento, ni los procesadores artesanales ni los responsables de la agroindustria pública, cursaron programas educativos dirigidos a obtener las destrezas y competencias técnicas requeridas afines al cultivo de aloe para la iniciativa a emprender, más allá de los programas de capacitación informal desarrollados por algunos entes ligados al cultivo en el estado. Sólo los em- 
prendedores de la agroindustria privada tuvieron formación para el emprendimiento, razón por la cual el sistema educativo tuvo poco o ningún efecto en la decisión de emprender. Los agentes entrevistados señalan la experiencia personal, conocimiento y cultura general adquiridos en el transcurso del tiempo, como su principal fortaleza valorada $(30 \%)$ a la hora de decidir emprender.

En este contexto, al identificar los emprendimientos más exitosos en términos de acceso a mercados y a tecnología, resalta la agroindustria privada, dada su mayor interacción con proveedores confiables y reconocidos, así como con clientes demandantes de sus productos, lo cual les permitió obtener información de primera mano para la acertada toma de decisiones. Esta productiva interacción también puede ejemplificarse a nivel de las asociaciones y cooperativas de productores, a través de la cercanía política con centros de decisión del Ejecutivo, lo cual les ha permitido acceso a programas de financiamiento para ampliar siembras y fondos de capital de trabajo (Piña et al., 2011a; 2011b).

De acuerdo con los aspectos descritos, el perfil tipo del emprendedor en el cultivo aloe, se corresponde con un hombre joven, con formación secundaria completa, de clase media baja, quien decidió incursionar en esta actividad para mejorar su situación económica y quien no ha recibido una formación especializada en el área del emprendimiento o en las destrezas técnicas para el procesamiento del aloe, y en su mayoría, no realizó un plan de negocios para orientar la concepción y puesta en marcha del emprendimiento.

\section{b. Perfil del emprendimiento en el cultivo aloe Falcón}

La siguiente categoría de análisis se centra en la actividad productiva desarrollada por el emprendedor, como resultado del aprovechamiento de una oportunidad dentro del cultivo aloe.

La puesta en marcha de los emprendimientos consultados se circunscribe posterior al año 2001 (53\%). Los más antiguos corresponden a los procesadores artesanales, atendiendo a la característica del paso generacional ya mencionado. Las asociaciones y cooperativas de productores se ubican alrededor del año 2000 y principio del 2006, mientras que la mediana empresa data del 2005. Por otro lado, sólo $60 \%$ de los consultados manifestó tener registro mercantil, lo cual se observa en la Tabla 2.

Los procesadores artesanales son los agentes donde éste aspecto es, en términos generales mayormente ignorado, precisamente por no necesitarlo para ninguna tramitación legal importante dentro de su proceso productivo. No lo requieren en ningún caso. Sus productos son informales y dirigidos a un nicho de mercado muy particular, para el cual éste, no es una exigencia determinante, al contrario, la clandestinidad del proceso le otorga cierto valor a los productos mercadeados. Se trata de un elemento distintivo de la actividad. Tal como se evidencia, los emprendimientos en el cultivo aloe tienen pocos años de vida.

Este rasgo determina el carácter de sector primario del aloe en Falcón. Si bien son muchos los intentos por materializar una iniciativa a nivel de procesamiento de bienes finales, es apenas unos diez (10) 
Tabla 2

Año de fundada, registro mercantil y empleos generados

\begin{tabular}{cccccc}
\hline \multicolumn{2}{c}{ Año de fundada } & \multicolumn{2}{c}{ Registro mercantil } & \multicolumn{2}{c}{ Empleos generados } \\
\hline $1968-1978$ & $7 \%$ & Si & $60 \%$ & $\begin{array}{c}\text { Inicio } \\
\text { (promedio) }\end{array}$ & 18 \\
$1979-1989$ & - & & & & \\
$1990-2000$ & $40 \%$ & No & $40 \%$ & Actual & 21 \\
$2001-2009$ & $53 \%$ & & & (promedio) & \\
\hline
\end{tabular}

Fuente: Elaboración propia.

años atrás cuando se comienza a contabilizar esas iniciativas de manera formal. Por tal razón, la inversión realizada es variada, oscilando desde 100 US \$ a 1,5 millón de US \$ (1 US \$ = 6,30 BsF), de acuerdo con los grupos considerados en la Tabla 3.

Éste rango tan disímil confirma una vez más la naturaleza de las iniciativas en el rubro aloe, en donde los procesadores artesanales realizan mínimas inversiones dado su modelo de negocio (Piña y González, 2010). En contraste, la agroindustria destinó recursos por el orden de los 1,5 millones de US \$. La inversión para las asociaciones y cooperativas de productores son un caso particular, dado que ellos ni como productores individuales ni como estructura asociativa, realizaron ninguna inversión. La construcción de las plantas, junto a aspectos de capacita- ción y organización, correspondieron a aportes del Ejecutivo Nacional y Regional. Este monto supera 2 millones US \$ por procesadora.

La orientación de la inversión en el caso de la agroindustria privada, se dirige al segmento de gel $1 \mathrm{X}$ y bienes finales tal como bebidas saborizadas. Los procesadores artesanales mantienen una línea de producción de medicamentos y cosméticos, principalmente, con escasa evolución en el tiempo y prácticamente nula incorporación de mejoras innovativas, tanto a nivel de proceso productivo como a la introducción de nuevos productos en el mercado. Para las asociaciones de productores y cooperativas, la concepción original era obtener aloína refinada y acíbar concentrado. En este sentido, los principales clientes de los emprendedores de aloe son básicamente agentes

Tabla 3

Inversión inicial, ventas iniciales y actuales

\begin{tabular}{lccc}
\hline \multicolumn{1}{c}{ Grupo } & $\begin{array}{c}\text { Inversión Inicial } \\
(\$)\end{array}$ & $\begin{array}{c}\text { Ventas Iníciales } \\
(\$ / \text { mes })\end{array}$ & $\begin{array}{c}\text { Ventas Actuales } \\
(\$ / \text { mes })\end{array}$ \\
\hline Artesanales & 100 & 2 & 250 \\
Agroindustria Privada & 1.500 .000 & 5.000 & 50.000 \\
Agroindustria Pública & 2.000 .000 & 40 & 150 \\
\hline
\end{tabular}

Fuente: Elaboración propia. 
intermediarios: cadenas de farmacia (agroindustria privada), otras procesadoras (asociación y cooperativas de productores), consumidores finales y tiendas naturistas (procesadores artesanales).

En otro orden ideas, resalta que estos emprendimientos no son intensivos en el uso de mano de obra, a diferencia de la producción primaria al momento de la cosecha y de la limpieza, por ejemplo, cuando concentran un significativo número de personal. En total, el número promedio de empleos generados está alrededor de 18 para el caso de la agroindustria pública y privada. Tal como ya se mencionó, los procesadores artesanales son en su mayoría, iniciativas unipersonales.

En cualquier caso, este número ha variado poco o nada desde la puesta en marcha de la iniciativa. En ocasiones aumentó 3 ó 4 nuevos empleos, pero no más allá de esa cifra. La mayor cantidad total para un momento particular, fue de 44 . En el caso de las asociaciones y cooperativas de productores, dadas sus características particulares regidas por ley, la cuantificación de empleos se realizó atendiendo a dicha normativa. Sin embargo, estas iniciativas no están en completa actividad y algunas están cerradas, por lo cual, su generación de empleos es nula.

Desde otro punto de vista, la información necesaria para iniciar el emprendimiento, tal como lo refleja la Tabla 4 provino en el caso de la mediana empresa, de un estudio de mercado llevado a cabo por sus socios, el cual sustentó el proyecto elaborado como requisito de sus estudios de fin de maestría. Por su parte, los procesadores artesanales nunca elaboraron un plan de negocios, mientras que las asociaciones y cooperativas de productores contaron con el proyecto técnico de la planta procesadora, donde se incluía un breve estudio de mercado y una proyección financiera general, en algunos casos, muy alejada de la realidad.

Este aspecto contribuye a fortalecer la tesis del significativo desconocimiento de la dinámica del aloe, por parte de la mayoría de los agentes del cultivo, principalmente productores y procesadores artesanales. Tal situación ha llevado a tomar decisiones sobre supuestos e informaciones no verificados, explicando en gran medida la actual situación exhibida por el cultivo en el estado Falcón.

Tabla 4

Origen de información, tiempo transcurrido y apoyo institucional

\begin{tabular}{lccccc}
\hline \multicolumn{2}{c}{ Origen información } & \multicolumn{2}{c}{ Tiempo transcurrido (años) } & \multicolumn{2}{c}{ Apoyo institucional } \\
\hline Pública & $20 \%$ & $1-5$ & 10 & Si & $75 \%$ \\
& & $5-10$ & 10 & & \\
Privada & $80 \%$ & $10-15$ & 60 & No & $25 \%$ \\
& $>15$ & 20 & & \\
\hline
\end{tabular}

Fuente: Elaboración propia. 
En otro orden de ideas, al consultarle a los emprendedores acerca del tiempo transcurrido desde que pensaron en iniciar su emprendimiento hasta el formal inicio del mismo, las respuestas fueron significativamente disimiles. Para algunos procesadores artesanales transcurrió desde menos de un año, hasta incluso diez (10) años, debido principalmente a la postergación de la decisión a emprender. Esperaban mejores condiciones del entorno para la puesta en marcha de su iniciativa.

La agroindustria privada tardó un año y medio, tiempo invertido en afinar detalles del plan de negocios, adquisición de equipos, contactar proveedores y clientes. Por su parte, algunas asociaciones y cooperativas de productores, aún no terminan de consolidar su emprendimiento. Del total de plantas procesadoras públicas previstas, todas están concluidas, sin embargo, debido a factores técnicos y de mercado, actualmente están paralizadas.

El financiamiento necesario para la puesta en marcha de los emprendimientos de las asociaciones y cooperativas de productores, provino fundamentalmente de fondos públicos. Para el caso de la agroindustria privada y los procesadores artesanales, fueron fondos propios. Es importante destacar que el rubro no figuraba hasta 2003 como objeto de crédito de las instituciones y organismos financistas del Estado, debido entre otros factores, a lo reciente de la actividad agroindustrial y a su significativo nivel de riesgo plasmado en el historial reciente del sector en Falcón, y en general, en el país. De esta manera, el emprendimiento promedio en el cultivo aloe se corresponde con aquella iniciativa registrada formalmente, con 5 años de fundada, genera alrededor de 20 empleos, con ventas actuales superiores a 50.000 US \$ y cuyos principales clientes son otras procesadores o intermediarios.

\section{c. Visión actual y futura del emprendimiento en el cultivo aloe Falcón}

En este acápite se desarrollan los planteamientos de los emprendedores respectos a la visión de la situación actual de sus emprendimientos, así como la proyección de los mismos en un horizonte de tiempo de corto y mediano plazo.

De tal análisis, el principal problema señalado por los emprendedores a la hora de constituir sus empresas, fue precisamente el acceso a capital (43\%), seguido de tecnología (27\%), mano de obra y adquisición de equipos con $10 \%$, cada uno. Estos porcentajes se entienden en el contexto del punto descrito anteriormente sobre el financiamiento: del total de entrevistados, fueron las asociaciones y cooperativas de productores para quienes el capital es su mayor limitante a la hora de poner en marcha su idea de emprendimiento, no tanto referido a la disponibilidad de capital en sí, sino en términos de su acceso y de lo dinámico y expedito de los procedimientos para su obtención, los cuales califican como de extremadamente engorrosos, lentos y burocráticos.

Llama la atención que los emprendedores entrevistados no señalaron 
como un grave problema o limitante de sus emprendimientos, aquellos referidos a la producción primaria del aloe (particularmente los asociados a enfermedades, las cuales han mermado significativamente los rendimientos). En efecto, estos agentes se enfocaron en el procesamiento agroindustrial y sus respuestas se centraron en su actuación como agroprocesadores y no como productores primarios.

Asimismo, sólo la agroindustria privada manifestó como uno de sus principales problemas, las condiciones macroeconómicas del país, las cuales les impedían continuar, de manera competitiva su proceso exportador, razón por la cual decidieron (en menos de 4 años de vida del emprendimiento), redimensionar y reorientar su modelo de negocio y dirigirlo hacia el mercado nacional con productos finales como bebidas saborizadas a base de aloe. Este producto resultó en una innovación dentro del segmento de bebidas y alimentos del país. Esta mención se corresponde perfectamente con el modelo de negocio de la agroindustria privada y el entorno donde ésta tiene oportunidad, evidenciando dos niveles o tipos de emprendimiento en el cultivo, moderadamente diferentes.

Por otro lado, a la hora de decidir poner en marcha su iniciativa, los emprendedores recurrieron en su totalidad a instituciones y organismos públicos para la búsqueda de información y asesoría a los fines de concretar la misma, destacando que si bien habían sido atendidos satisfactoriamente, no obtuvieron la totalidad de la información solicitada, obligándolos a recurrir a otras fuentes de in- formación, principalmente de carácter privado, tales como firmas consultoras y de asesoría. En este contexto, $45 \%$ de los entrevistados se muestran muy optimistas y visualizan su emprendimiento creciendo y diversificando su producción. En contraste, $15 \%$ ve su emprendimiento igual y $5 \%$ lo visualiza cerrado.

\section{Dinámica emprendedora en el cultivo aloe}

A fin de contextualizar el análisis de la dinámica emprendedora del aloe en Falcón, es importante iniciar con el origen del cultivo en el estado. Sobre el particular, los referentes históricos ratifican como coherente la versión de la entrada de la planta a la región desde las Antillas, luego de llegar a éstas desde las Islas Canarias $u$ otra isla del Caribe, en alguno de los distintos viajes emprendidos por los navegantes europeos.

Específicamente, en Falcón existe evidencia desde 1790 del uso del aloe en el estado (Madriz, 1790), época desde la cual su esquema productivo se ha enfocado en la recolección del acíbar para su exportación, principalmente a Europa como mercado original y tradicional. En otras palabras, en Falcón sólo se realiza la adecuación necesaria para permitir el traslado del producto a los centros de transformación y consumo, siendo éste, el origen de la pasta de aloe.

En términos concretos, el estado Falcón ha funcionado como enclave de producción de un commodity, el cual siempre fue manejado como un cultivo de recolección y es apenas a partir de principios de la década del '90, cuando comien- 
za su evolución hacia un modo de explotación y producción más cercana a esquemas propios de cultivos tradicionales, con un fin más industrial para satisfacer nichos de mercado, en respuesta a su significativo crecimiento y especialización en los últimos años.

Es así como, en respuesta a las oportunidades de inversión derivadas de dicha especialización y crecimiento exhibido por el aloe, los agentes locales vieron en estos nichos una importante oportunidad de emprendimiento, pero tal proceso evolutivo se encontró en Falcón con dos importantes escollos: mercado y tecnología. En primer lugar, el aloe es un cultivo de carácter agroindustrial, no es principalmente agroalimentario, en consecuencia, sus fines se orientan hacia la producción de bienes intermedios diferenciados dirigidos a la industria cosmética, farmacéutica o médica, la cual exige cumplir con significativos protocolos de calidad, que los tradicionales esquemas de producción aplicados históricamente en el estado, están lejos de satisfacer.

De esta manera, al evaluar las opciones para entrar competitiva y eficientemente en el mercado del aloe, el sector primario del estado se encuentra con la imposibilidad de igualar los estándar de calidad exigidos internacionalmente, esto ligado de manera estrecha, al acceso y operación de la tecnología requerida para tal transformación. Por otro lado, se enfrenta a un mercado dominado por transnacionales del aloe, las cuales mantienen el control de la provisión de insumos y de los canales de distribución de los bienes finales. Adicionalmente, el entorno institucional público oficial, no contemplaba al aloe como un rubro prioritario, siendo apenas en 2004, con la promulgación del decreto 363 cuando comienzan los primeros pasos en este sentido.

Tal panorama configura un entorno muy poco propicio para el surgimiento de iniciativas emprendedoras asociadas a la transformación del aloe, signadas de manera particular y como era de esperarse, por la ausencia de un marco institucional favorable de tales iniciativas, en donde la participación de colectivos de productores, se enfocó en la búsqueda de soluciones en el sector oficial, dejando de lado la iniciativa propia y dando pie a un abrigo paternalista el cual ha sabido capitalizar políticamente esa carencia y transformarla en una importante fuente de recursos financieros destinados a impactar en forma descoordinada e incoherente sobre un cultivo del cual conoce poco acerca de su dinámica y comportamiento. En términos concretos, el Ejecutivo, tanto nacional y regional, han invertido recursos superiores a los 15 millones de US \$ entre 2004 y 2009.

Aunado a lo anterior, la dinámica emprendedora del aloe en Falcón está fuertemente asociada al esquema de producción del cultivo a nivel mundial, principalmente orientado a la obtención de hojas para la elaboración de gel $1 \mathrm{X}$, dirigido a la industria cosmética, médica y alimentaria, las cuales poseen altos estándares de calidad y exigen estrictos protocolos de manipulación de dicha materia prima. Esta orientación hacia la elaboración del gel requiere de la provisión de hojas con un mínimo de tamaño y peso, principalmente por razones de costos, y por ende, de rentabilidad, lo cual a su vez implica el 
uso de importantes volúmenes de agua para el riego de las plantaciones.

Sobre este particular tema del agua, Falcón tiene fuertes limitaciones para la producción de hojas bajo riego, principalmente debido a la disponibilidad y acceso a fuentes para regar, a una alta inversión inicial para la instalación del sistema de regadío y a los bajos precios relativos pagados por kilogramo de hoja, por lo cual la racionalidad económica de los productores se orienta y se ha orientado a lo largo de los años, a la explotación de aloe para acíbar, destinando el riego para la siembra de otros rubros de mayor retorno en menor plazo de espera. Complementariamente, es importante destacar cómo los modelos de negocio afines al aloe en Falcón, asociados a la producción de gel y sujetos a una significativa inversión inicial, responden a esos segmentos dominados por trasnacionales y su dominio de los canales de distribución.

Asimismo, la puesta en marcha de emprendimientos de la naturaleza descrita, implica el uso de componentes tecnológicos los cuales requieren de destrezas y competencias específicas de manejo y han descartado casi por completo al acíbar y su transformación, el cual también posee amplias posibilidades de uso en sectores industriales como pinturas y recientemente, la construcción, los cuales se encuentran incipientemente explorados y abordados por nuevos emprendimientos y es la vocación natural del estado.

Atendiendo a la evidencia anterior y al aplicar un análisis schumpeteriano al tipo de emprendimiento presente en el aloe, destaca la ausencia de innovación y la creatividad, resaltando modelos de imitación, debido ya sea a la falta de un fuerte apoyo de los centros I/D locales o del fomento de los procesos innovadores a través de la interacción entre agentes próximos a las actividades afines al cultivo. En cuanto a la actuación de los centros, son significativas las limitaciones de recursos y lo disperso de sus acciones y planes, limitando así el apoyo requerido.

Como resultado de lo anterior, los escasos emprendimientos materializados en el cultivo aloe, corresponden a imitaciones de experiencias ya existentes, con bajo o nulo porcentaje de innovación en el modelo de negocio implementado. En tal contexto, el emprendimiento se corresponde con la imitación y no con la innovación, lo cual le ha restado competitividad para posicionarse en un nicho específico de mercado. Los emprendimientos no tienen poder para competir con empresas tradicionales, establecidas y con dominio del mercado.

Por otro lado, desde una perspectiva kirzneriana del individuo atento a las variaciones del mercado, es importante diferenciar y manejar el conocimiento en contraste a la información. La información posibilita a los agentes del cultivo tener una visión general de los hechos y fenómenos que están ocurriendo en torno a esta planta, pero sin suministrarle los detalles básicos y esenciales de cómo están ocurriendo. Es sólo cultura general. Por su parte, el conocimiento otorga los medios para potencialmente consolidar un emprendimiento en términos de comportamiento del mercado, las variaciones y adecuaciones competitivas del proceso productivo. El dominio del conocimiento 
es un proceso costoso y no pocas veces, lento y de difícil acceso.

En tales condiciones, los agentes del cultivo aloe en Falcón manejan un significativo alto volumen de información, el cual en ciertas ocasiones ha llegado a confundir y dispersar esfuerzos, pero en contraste, tienen a su disposición un muy bajo nivel de conocimiento acerca del rubro y su comportamiento orientado a identificar y consecuentemente, aprovechar oportunidades de emprendimiento. De esta manera se evidencia la ruptura entre generación y difusión de conocimiento con el proceso emprendedor.

Bajo tal panorama, destacan dos hechos concretos y particulares: dada la ausencia de información apropiada acerca de las reales potencialidades del aloe en Falcón en términos de sus características climáticas y su natural condición para el acíbar, se ha orientado su desarrollo hacia el modelo de producción de gel predominante en el escenario mundial, enfatizando cursos de acción hacia un esquema productivo primario con fuertes restricciones en el estado.

Por otro lado, un porcentaje significativo de generación de conocimiento se ha orientado al aprovechamiento de las resinas como compuestos anticorrosivos, con muchas posibilidades de aplicación en la industria de pinturas, por ejemplo, a diferencia del escaso conocimiento generado en torno al gel más allá de los productos tradicionales sin mayor innovación en los mismos, elaborados por procesadores artesanales y algunas iniciativas farmacéuticas (Piña y González, 2010).

En función de lo anterior, el emprendimiento en el cultivo aloe en Falcón debe ser el resultado de factores de orden interno y de orden externo. Desde el punto de vista interno, destaca el emprendedor: individuo en donde se materializan a través de su formación, destrezas-habilidades necesarias para iniciar y adelantar este proceso; la experiencia: asociada a los esquemas cognitivos presentes en el emprendedor para estimular y motivar su actuación en el emprendimiento; y la tecnología: competencias propias requeridas para el manejo del proceso productivo inherente, no sólo a la agrotransformación del aloe, sino a la innovación y mejora de bienes y servicios ya existentes en el mercado. La convergencia de estos tres factores deriva en el aprovechamiento de una oportunidad para el emprendimiento.

Por su parte, el componente externo está asociado al ámbito de dónde surgen esas oportunidades, ya sean creadas (Schumpeter) o existentes (Kirzner), lo cual está asociado fuertemente a la matriz institucional en torno al cultivo aloe junto a la manera cómo ocurre la difusión del conocimiento asociado al capital social de los agentes del cultivo, en tanto las redes de actores interconectados bajo una visión compartida, un argumento vertebrador. Sin embargo, los resultados de la investigación evidencian un perfil institucional en el cultivo aloe, igualmente, poco favorable para el emprendimiento, principalmente debido a elementos de tipo estructural, tal como factores de orden macroeconómico (diferencial cambiario), operativos (infraestructura) y mecanismos de apoyo o legales (reglamentos y leyes).

Igualmente existen aspectos de tipo cognitivo, reflejado en el rol de los 
responsables de las agroindustrias públicas, quienes manifestaron carecer de las competencias y destrezas necesarias para iniciar actualmente un exitoso proceso de manufactura y exportación de los bienes a generar. Así, la ausencia de emprendimientos en el cultivo aloe descansa más en la falta de incentivos y estímulos para el surgimiento de dichas iniciativas, junto a una normativa favorable promotora del desarrollo de competencias y destrezas en los sujetos participantes, en contraste a la falta de oportunidades de inversión en el sector, el cual exhibe hoy día una interesante dinámica con varias opciones de negocio.

\section{Reflexiones finales}

La dinámica emprendedora en el cultivo aloe en el estado Falcón, aparece como limitada y concentrada a nivel de producción primaria. Son escasas las iniciativas en el ámbito agroindustrial y menos aún, en la fase de elaboración de bienes finales donde sólo aparecen procesadores artesanales y fundamentalmente, con emprendimientos informales caracterizados por la gestión de un conocimiento ancestral pasado de generación en generación y en donde no se aprecia un sistemático y planificado proceso productivo.

Por su parte, las asociaciones y cooperativas de productores han visto en los programas de financiamiento y desarrollo de infraestructura del Ejecutivo Nacional y Regional, la posibilidad de concretar sus intenciones de emprendimiento, frustrados en varias oportunidades debido principalmente, al modelo de nego- cio presente en el cultivo aloe, así como al marco institucional derivado del mismo.

En consecuencia, se consolidó un modelo de negocio de tipo extractivo, sin las fases siguientes de transformación y conexión con el mercado. Estos elementos han conllevado a la especialización de los agentes en la fase primaria del cultivo (siembra - cosecha) y han obviado el procesamiento agroindustrial, la elaboración de bienes finales y la creación del andamiaje institucional necesario para promover iniciativas de emprendimiento en las áreas de procesamiento y manufactura.

En cierta medida, ese modelo de negocio no ha creado el ambiente (entorno) ni las capacidades y destrezas para abordar el mercado de consumidores finales. De esta manera, hoy día resulta significativamente cuesta arriba, en términos de costo, tiempo y recurso humano, adelantar un programa acorde a las exigencias del momento. La acción del Ejecutivo se ha centrado los últimos años en la construcción de infraestructuras para el procesamiento de acíbar y la ampliación de la superficie de siembra, sin embargo, se ha descuidado significativamente el aspecto tecnológico y la conexión de éste con el mercado. La estrategia se ha enfocado desde el lado de la oferta, desconociendo casi por completo las características y particularidades de la demanda, quien en definitiva remunera el bien elaborado.

En este punto es importante destacar el preocupante desconocimiento por parte de la mayoría de los agentes del cultivo, de las características del mercado del aloe, ya sea nacional y mucho 
menos internacional. Es realmente sorprendente cómo se ha actuado casi a ciegas dentro del sector. En tal sentido, se impone la actuación en varios frentes y varios niveles. El primero corresponde al Ejecutivo como garante de las reglas de juego, se considera que es el actor llamado a brindar esas condiciones mínimas para el emprendimiento en el cultivo aloe, en términos de facilitar y simplificar los trámites para la formalización de iniciativas de emprendimiento, así como la provisión de información acerca del comportamiento del sector a nivel nacional e internacional, entre otros aspectos.

Asimismo, la promoción y desarrollo de actividades de innovación y desarrollo tecnológico, en el amplio espectro de aplicaciones que presenta el aloe, es otro de los aspectos a abordar en el cultivo. Hoy día, el desempeño y crecimiento del sector aloe está determinando, precisamente por el nivel de desarrollo tecnológico y el lanzamiento al mercado de nuevos productos cada vez con mayor valor agregado, producto de intensos programas de investigación aplicados por las principales empresas del ramo. Por otro lado, debe tenerse muy en claro que sin el real conocimiento del mercado, todos los intentos de emprendimiento resultan innecesarios, ya que no existiría la lógica conexión entre oferta y demanda y será un riesgo extremo la inversión de esfuerzos y capital en tales circunstancias.

\section{Referencias Bibliográficas}

Grebel, Thomas; Pyka, Andreas y Hanusch, Horst (2001). An evolutionary approach to the theory of entrepreneurship. Discussion Paper Series
206, Institute for Economics, Universitaet Augsburg.

Jack, Sarah y Anderson, Alistair (2002). The effects of embeddedness on the entrepreneurial process. Journal of Business Venturing, 17: 467-487.

Kantis, Hugo; Angelelli, Pablo y Moori Koenig (2004). Desarrollo emprendedor: América Latina y la experiencia internacional. Santiago de Chile: Banco Interamericano de Desarrollo y Fundes Internacional. 354 pp.

Kirzner, Israel (1997). Entrepreneurial discovery and the competitive market process: an austrian approach. Journal of Economic Literature, 35 (1): 60-85.

Madriz, Juan (1790). Testamentarias. Archivo Histórico del Estado Falcón. UNEFM. Sección Testamentarías, Caja 58. Folio 46-46v.

Marshall, Alfred (1920). Principles of Economics. Londres: Macmillan and Co., Ltd. 8th edition.

Montoya, Omar (2004). Schumpeter, innovación y determinismo tecnológico. Scientia et Technica, 25: 209-213.

North, Douglas (1991). Institutions. Journal of Economic Perspectives, 5 (1): 97112.

Park, John (2005). Opportunity recognition and product innovation in entrepreneurial hi-tech start-ups: perspective and supporting case study. Technovation, 25: 739-752.

Piña, Henri; Tua, Juan y Morales, Agustín (2011a). Capital social en la cadena aloe, estado Falcón. Venezuela, Cuadernos de Desarrollo Rural, 8 (66): 103-122.

Piña, Henri; Franquiz, Joglen y Morales, Agustín (2011b). Estructura, función y jerarquía de actores en la cadena aloe en el estado Falcón, Venezuela. Agricultura, Sociedad y Desarrollo, 8 (2): 209-227. 
Piña, Henri y Morales, Agustín (2010). Aloe en Venezuela: de la cadena de valor al distrito industrial. Revista Problemas del Desarrollo, 41 (160): 187208.

Piña, Henri y González, Joirabid (2010). La agroindustria en la cadena zábila en el estado Falcón, Venezuela. Revista Multiciencias, 10 (1): 13-20.

Piña, Henri y Chirino, Lusben (2008). Mercado de la zábila (Aloe vera L.) en el estado Falcón. Revista de la Facultad de Agronomía LUZ, 25: 364-392.

Piña, Henri (2006). El circuito zábila (Aloe vera L.) en el estado Falcón, Venezuela. En A. Álvarez, F. Boucher, F. Cervantes, A. Espinoza, J. Muchnik. \& D. Requier, Agroindustria rural y territorio, tomo I: los desafíos de los sistemas agroalimentarios localizados (págs. 229-254). México: Universidad Autónoma del Estado de México. $445 \mathrm{pp}$.

Piña, Henri (2005). Perfil preliminar del mercado de la zábila (Aloe barbadensis Mill.) en el estado Falcón, Venezuela. BIOAGRO, 17 (2): 85-92.

Piña, Henri (2004). El conglomerado zábila (Aloe vera L.) en el estado Falcón, Venezuela. Cuadernos de Desarrollo Rural, 53: 37-57.

Plummer, Lawrence; Haynie, John y Godesiabois, Joy (2007). An essay on the origins of entrepreneurial opportunity. Small Business Economics 28: 363-379.

Presutti, Manuela y Boari, Cristina (2008). Space - related antecedents of social capital: some empirical inquiries about the creation of new firms. International Entrepreuner Management Journal, 4: 217-234.

Romero, Ana y Montoro, Ángeles (2008). How clusters can encourage entrepreneurship and venture creation. Reasons and advantages. International Entrepreuner Management Journal, 4: 315-329.

Schumpeter, Joseph (2003). Entrepreuner. Advances Austrian Economics, 6: 235-265

Schumpeter, Joseph (1947). Creative response economic history. The Journal of Economic History, 7 (2): 149-159.

Schumpeter, Joseph (1927). The explanation business cycle. Economica, 21: 286-311.

Shane, Scott y Venkataraman, Sheila (2000). The promise of entrepreneurship as a field of research. The Academy of Management Review, 25 (1): 217-226.

Sorenson, Oskar y Audia, Phillipe (2000). The social structure of entrepreneurial activily: geographic concentration of footwear production in the United States, 1940-1989. American Journal of Sociology, 106 (2): 424-462.

Tíryakí, Ahmet (2008). Theories of Entrepreneurship: A Critical Overview. Ýktisat: Anadolu Üniiversitesi. 12 pp.

Vaghely, Ivan y Julien, Pierre (2010). Are opportunities recognized or constructed?: An information perspective on entrepreneurial opportunity identification. Journal of Business Venturing, 25 (1): 73-86. 